\title{
The classic prognostic factors in advanced Hodgkin's lymphoma patients are losing their meaning at the time of Pet-guided treatments
}

\author{
Alessia Bari ${ }^{1} \cdot$ Raffaella Marcheselli ${ }^{2}$. Stefano Sacchi ${ }^{3} \cdot$ Alessandro Re $^{4} \cdot$ Chiara Pagani $^{4} \cdot$ Alessandra Tucci $^{4}$. \\ Barbara Botto $^{5}$. Umberto Vitolo ${ }^{5}$. Anna Lia Molinari ${ }^{6}$ - Benedetta Puccini ${ }^{7}$ - Alessandro Pulsoni ${ }^{8}$. \\ Armando Santoro ${ }^{9,10}$. Monica Tani ${ }^{11}$. Luca Nassi $^{12}$. Erika Meli ${ }^{13}$ - Vincenzo Pavone ${ }^{14}$ Maurizio Bonfichi $^{15}$. $^{2}$ \\ Andrea Evangelista ${ }^{16}$. Daniela Gioia ${ }^{2}$ - Alessandro Levis ${ }^{2}$. Pierluigi Zinzani ${ }^{17}$ • Fondazione Italiana Linfomi (Onlus)
}

Received: 8 April 2019 / Accepted: 6 December 2019

(C) The Author(s) 2019, corrected publication 2020

\begin{abstract}
The International Prognostic Score (IPS) is the most commonly used risk stratification tool for patients with advanced Hodgkin lymphoma (HL). It incorporates seven clinical parameters independently associated with a poorer outcome: male sex, age, stage IV, hemoglobin level, white blood cell and lymphocyte counts, and albumin level. Since the development of the IPS, there have been significant advances in therapy and supportive care. Recent studies suggest that the IPS is less discriminating due to improved outcomes with ABVD therapy. The aim of the present study was to asses if classic prognostic factors maintain their prognostic meaning at the time of response-adapted treatment based on interim PET scans. We evaluated the prognostic significance of IPS in the 520 advanced stage HL patients enrolled in the PET-guided, HD0801 trial in which PET2-positive patients underwent a more intense treatment with an early stem-cell transplantation after 2 cycles of ABVD. We observed that in these patients, the IPS completely loses its prognostic value together with all the single parameters that contribute to the IPS. Furthermore, neutrophils, monocytes, lymphocytes, and the ratio among them also no longer had any predictive value. We believe that the substantial improvement in survival outcomes in PET2-positive patients treated with early autologous transplantation could explain the complete disappearance of the residual prognostic significance of the IPS.
\end{abstract}

Alessia Bari and Raffaella Marcheselli contributed equally to this work.

Stefano Sacchi

stefano.sacchi@unimore.it

1 UO Terapie Mirate in Oncoematologia ed Osteoncologia, Dipartimento di Scienze Mediche e Chirurgiche Materno-Infantili e dell'Adulto, Universita' di Modena e Reggio Emilia, Modena, Italy

2 Fondazione Italiana Linfomi, Onlus, Alessandria, Italy

3 UO Terapie Mirate in Oncoematologia ed Osteoncologia, Dipartimento di Scienze Mediche e Chirurgiche Materno-Infantili e dell'Adulto, Universita' di Modena e Reggio Emilia, Modena, Italy

4 SC Ematologia ASST Spedali Civili, Brescia, Italy

5 Struttura Complessa Ematologia, AOU Città della salute e della scienza di Torino, Turin, Italy

6 Unità Operativa di Ematologia, Ospedale degli Infermi di Rimini, Rimini, Italy

7 SOD Ematologia AOU Careggi, Florence, Italy

8 Dipartimento di Biotecnologie Cellulari ed Ematologia, Sapienza Università di Roma, Rome, Italy
9 Humanitas Clinical and Research Center - IRCCS, Humanitas Cancer Center, via Manzoni 56, 20089 Rozzano, Milan, Italy

10 Humanitas University, Department of Biomedical Sciences, Via Rita Levi Montalcini 4, 20090 Pieve Emanuele - Milan, Italy

11 U.O.C di Ematologia Ospedale Santa Maria delle Croci, Ravenna, Italy

12 Department of Translational Medicine, Università del Piemonte Orientale Amedeo Avogadro, Azienda Ospedaliero-Universitaria Maggiore della Carità, 28100 Novara, Italy

13 Ematologia, ASST Grande Ospedale Metropolitano Niguarda, Milan, Italy

14 A.O. C. Panico-U.O.C Ematologia e Trapianto, Tricase, Lecce, Italy

15 Div. di Ematologia, IRCCS Policlinico S. Matteo di Pavia, Pavia, Italy

16 Unit of Clinical Epidemiology, AOU Citta' della Salute e della Scienza di Torino and CPO Piemonte, Turin, Italy

17 Policlinico S.Orsola-Malpighi, Istituto di Ematologia "Seragnoli", Bologna, Italy 


\section{Indroduction}

For several years, the most widely utilized risk stratification tool for advanced Hodgkin's lymphoma (HL) patients has been the International Prognostic Score (IPS). It is a retrospectively developed clinical model based on the outcome of about 1600 patients with advanced stage disease. A large majority of these patients were treated before 1992 with a doxorubicin-containing regimen, while $20 \%$ received mechlorethamine, oncovin, procarbazine, and prednisone (MOPP) or similar regimens. By multivariate analysis, seven clinical parameters were independently associated with a poorer outcome: male sex, age $>45$ years, clinical stage IV, hemoglobin $<10.5 \mathrm{~g} / \mathrm{dl}$, WBC count $>$ $15 \times 10^{9} / \mathrm{L}$, lymphocyte count $<0.6 \times 10^{9} / \mathrm{L}$ or $8 \%$ of differential, and albumin $<4 \mathrm{~g} / \mathrm{dL}$ [1]. In 2012, Moccia et al. [2] evaluated individual IPS factors in 740 patients with stage III/IV or stage I/II HL who had B symptoms or bulky disease and were treated with adriamycin, bleomycin, vinblastine, and dacarbazine (ABVD) or an ABVD-equivalent regimen with curative intent. In an analysis restricted to 686 patients aged $\leq$ 65 years, as in the original index, the IPS remained prognostic for failure-free survival (FFS) and overall survival (OS), but with a narrower range of outcomes, probably related to the improvement in survival outcomes in all risk groups and mainly in the poorer-risk groups. Further, the results showed that all individual factors, with the exception of gender, were prognostic in univariate analysis for FFS, but only age and hemoglobin level maintained significance in multivariate analysis. These results confirmed that IPS remains prognostic in patients with advanced-stage $\mathrm{HL}$, but the range of outcomes delineated on the basis of the number of factors present at diagnosis has significantly diminished. To assess the utility of the individual IPS factors in the contemporary era, Diefenbach et al. [3] analyzed data from 854 patients with HL enrolled in the North American Intergroup trial, E2496 [4]. The results of the study showed that the IPS remained prognostic, but the separation between IPS groups narrowed, confirming the results from Moccia et al. [2]. Thus, they proposed an alternative prognostic index, the IPS-3, based only on 3 factors, age, stage, and hemoglobin level, showing that this model outperformed the original IPS on risk prediction for both FFS and OS. The aim of the present study was to assess whether classic prognostic factors maintain their prognostic meaning at the time of PET-guided treatment.

\section{Material and methods}

The HD0801 multicenter study [5] involved 520 patients with histologically documented, advanced-stage HL (clinical stage II B-IV) enrolled between September 2008 and April 2013 at 28
Italian centers. All patients received initial treatment with ABVD. After 2 cycles of ABVD, an interim PET was performed and 510 patients continued therapy according to the experimental protocol. Patient showing negative PET after 2 ABVD cycles (IPET or PET2) recieved an additional 4 cycles of ABVD, while patients who were I-PET-positive underwent an early intensification with 4 cycles of ifosfamide, gemcitabine, and vinorelbine (IGEV) followed by carmustine, cytarabine, etoposide, and melphalan (BEAM)-conditioned autologous bone marrow transplantation. All local ethic committees at each center approved the study protocol and its amendments in accordance with Italian law and in compliance with the Declaration of Helsinki. Patients provided written informed consent before being included in the study. Absolute monocyte count (AMC) was not included in the original electronic case report form (CRF), and 19 centers agreed to add $\mathrm{AMC}$ to the initial patient $\mathrm{CRFs}$

\section{Statistical analysis}

Progression-free survival (PFS) was defined as the time from I-PET to the time of any documented progressive disease, relapse, or death from any cause. Patient baseline characteristics are expressed as absolute frequencies and percentages for categorical variables. Continuous variables were reported as the median and 2.5-97.5 percentiles. The Kaplan-Meier method was used to estimate PFS. Statistical comparisons by groups of risk were performed with the log-rank test. As cutoffs for AMC, neutrophil lymphocyte ratio (NLR) and lymphocyte monocyte ratio (LMR), we utilized $0.75 \times 10^{9} / \mathrm{L}, 6$, and 2.1 , respectively, as previously published $[6,7]$.

\section{Results}

The median age of the patients was 33 years and 54\% were male. Most of the patients $(73 \%)$ had a nodular sclerosis subtype, and $46 \%$ presented with clinical stage IV disease. An IPS $\geq 3$ was observed in $43 \%$ of patients. The demographic and baseline disease characteristics of the entire population are listed in Table 1. After a median follow-up of 45 months, the Kaplan-Meier estimates for the entire population were $97 \%$ and $80 \%$ for OS and PFS, respectively. PET2-negative and PET2-positive patients had a PFS of $81 \%$ and $74 \%$, respectively. Overall, 97 patients underwent disease progression, 21 and 73 in the PET2 positive and negative group, respectively, and 18 patients died, 8 and 10 in PET2-positive and PET2negative group, respectively. 
Table 1 Baseline characteristics of the 510 patients who continued with the experimental protocol after I-PET

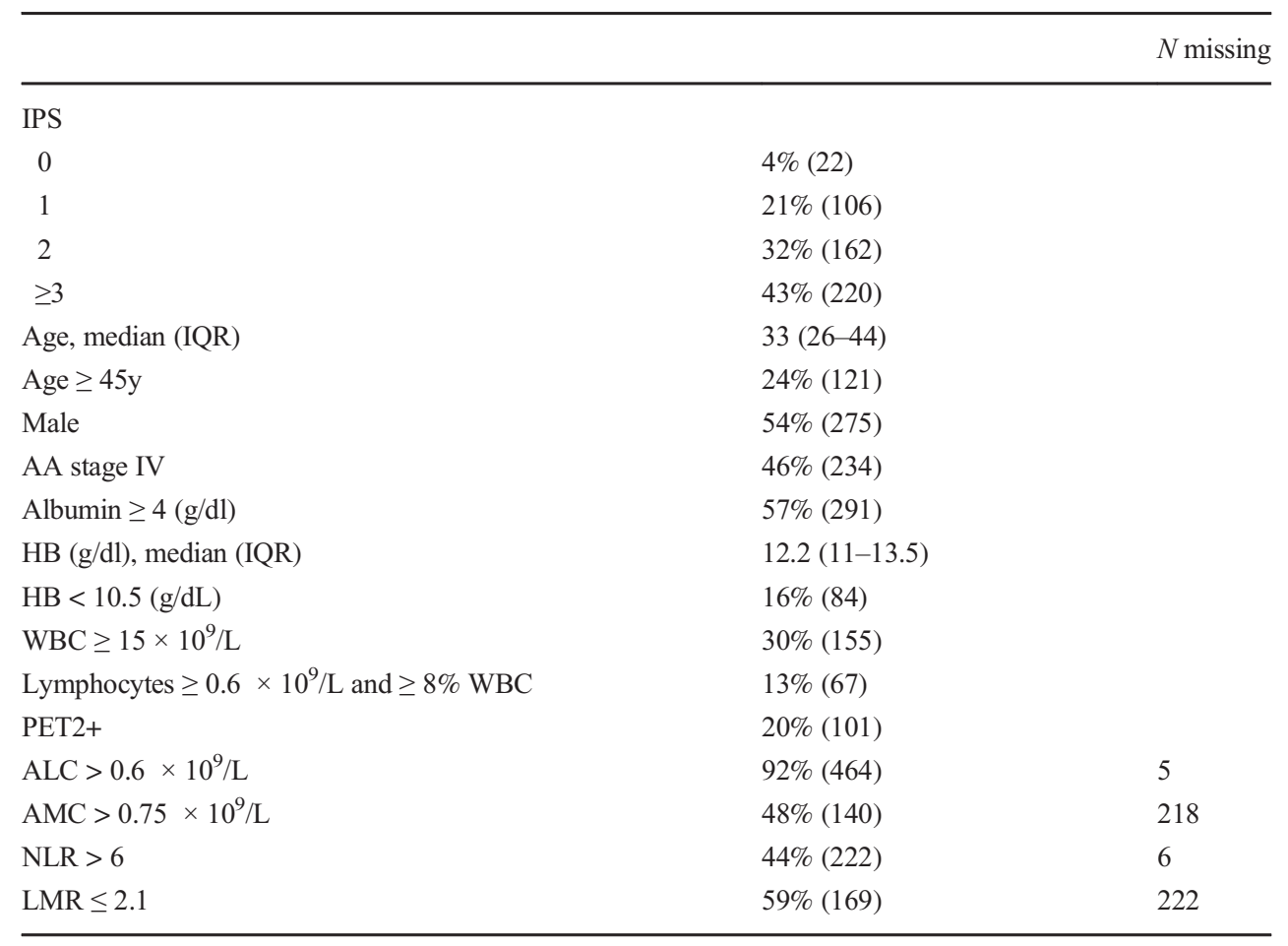

$A A$, Ann Arbour; $H B$, hemoglobin; $W B C$, white blood cells; $A L C$, absolute lymphocyte count; $A M C$, absolute monocyte count; $N L R$, neutrophil lymphocyte ratio; $L M R$, lymphocyte monocyte ratio

\section{Outcome according to IPS and single factors contributing to IPS}

We analyzed separately all patients, PET2-negative patients, and PET2-positive patients (Table 2). In the overall patient population, PFS according to IPS factors did not show statistically significant differences related to IPS score nor to single factors contributing to the IPS except for a significant difference related to hemoglobin level $\geq 10.5 \mathrm{~g} / \mathrm{dL}$ observed in all patients $(P=0.033)$ and in PET2-negative patients $(P=$ 0.003 ), and related to low lymphocyte counts in PET2positive patients $(P=0.032)$. Instead, analyzing PET2positive patients, not even hemoglobin values $<10.5 \mathrm{~g} / \mathrm{dL}$, discriminated patients with a worse PFS.

\section{Outcome according to AMC, NLR, and LMR}

In the 292 patients in which AMC was added to the original CFR, we also evaluated the prognostic meaning of AMC, NLR, and LMR. No statistically significant differences in PFS were observed between 234 patients $(80 \%)$ with a negative I-PET and 58 patients $(20 \%)$ with positive I-PET $(P=$ $0.260)$. In the overall population, patients with $\mathrm{AMC} \leq$ or $>$ $0.75 \times 10^{9} / \mathrm{L}, \mathrm{NLR}<6$ or $\geq 6$, and LMR $\leq 2.1$ or $>2.1$ had similar PFS, with no significant differences between groups (Table 3). Also, considering I-PET-positive and I-PETnegative patients separately, AMC, NLR, and LMR did not show any predictive effect for PFS. Further, AMC $\leq$ or $>0.75$ $\times 10^{9} / \mathrm{L}, \mathrm{NLR}<6$ or $\geq 6$, and $\mathrm{LMR} \leq 2.1$ or $>2.1$ at diagnosis were not associated with I-PET results. In addition, different AMC, NLR, and LMR cutoff points did not discriminate different prognostic groups in this series.

\section{Discussion}

In the modern era, the classic IPS retains its prognostic capacity in patients with advanced HL treated with ABVD. However, the range of outcomes has considerably narrowed $[2,3]$. This is probably related to improvement in survival outcomes in high-risk patients. Several reasons determine this improvement, including the enhanced diagnostic accuracy, and the large availability of better supportive care and neutrophil growth factors. To explore whether classic prognostic factors retain their meaning at the time of PET-guided treatment, we analyzed the recently published literature on this topic. The US Intergroup Trial of response-adapted therapy [8] enrolled 358 patients with stage III and IV HL in a phase II clinical trial. A PET scan (PET2) was performed after two courses of ABVD. Patients with a Deauville score 1 to 3 (PET2-negative) received an additional 4 ABVD cycles, while patients with a Deauville score of 4 and 5 (PET2positive) were switched to an intensified regimen of bleomycin, etoposide, doxorubicin, cyclophosmamide, 
Table 2 Kaplan-Maier estimates of PFS from PET2 according to IPS components.

\begin{tabular}{|c|c|c|c|c|c|c|c|c|c|c|}
\hline & & \multicolumn{3}{|c|}{ All patients } & \multicolumn{3}{|c|}{ PET2- } & \multicolumn{3}{|c|}{ PET2+ } \\
\hline & & $N$ & $\begin{array}{l}24- \\
\text { month } \\
\text { PFS }\end{array}$ & $P$ & $N$ & $\begin{array}{l}24- \\
\text { month } \\
\text { PFS }\end{array}$ & $P$ & $N$ & $\begin{array}{l}24- \\
\text { month } \\
\text { PFS }\end{array}$ & $P$ \\
\hline \multirow[t]{4}{*}{ IPS } & 0 & 22 & $90.5 \%$ & 0.257 & 19 & $94.4 \%$ & 0.118 & 3 & $66.7 \%$ & 0.490 \\
\hline & 1 & 106 & $79.6 \%$ & & 84 & $80.6 \%$ & & 22 & $75.0 \%$ & \\
\hline & 2 & 162 & $80.9 \%$ & & 130 & $83.5 \%$ & & 32 & $71.3 \%$ & \\
\hline & $\geq 3$ & 220 & $77.6 \%$ & & 176 & $77.1 \%$ & & 44 & $79.1 \%$ & \\
\hline \multirow[t]{2}{*}{ Age } & $<45$ & 389 & $80.2 \%$ & 0.118 & 305 & $81.6 \%$ & 0.054 & 84 & $75.3 \%$ & 0.888 \\
\hline & $\geq 45$ & 121 & $77.6 \%$ & & 104 & $77.8 \%$ & & 17 & $76.5 \%$ & \\
\hline \multirow[t]{2}{*}{ Gender } & Female & 235 & $78.7 \%$ & 0.770 & 187 & $80.3 \%$ & 0.896 & 48 & $72.6 \%$ & 0.715 \\
\hline & Male & 275 & $80.4 \%$ & & 222 & $80.9 \%$ & & 53 & $78.2 \%$ & \\
\hline \multirow{2}{*}{$\begin{array}{l}\text { Ann Arbor } \\
\text { stage }\end{array}$} & $<\mathrm{IV}$ & 276 & $79.7 \%$ & 0.356 & 225 & $82.1 \%$ & 0.213 & 51 & $69.5 \%$ & 0.551 \\
\hline & IV & 234 & $79.5 \%$ & & 184 & $78.9 \%$ & & 50 & $81.6 \%$ & \\
\hline \multirow[t]{2}{*}{ Albumin } & $\geq 4(\mathrm{~g} / \mathrm{dL})$ & 219 & $82.3 \%$ & 0.252 & 169 & $83.1 \%$ & 0.275 & 50 & $79.6 \%$ & 0.555 \\
\hline & $<4(\mathrm{~g} / \mathrm{dL})$ & 291 & $77.6 \%$ & & 240 & $78.9 \%$ & & 51 & $71.3 \%$ & \\
\hline \multirow[t]{2}{*}{$\mathrm{HB}$} & $\begin{array}{l}\geq 10.5 \\
(\mathrm{~g} / \mathrm{dL})\end{array}$ & 426 & $80.8 \%$ & 0.033 & 340 & $82.7 \%$ & 0.003 & 86 & $73.5 \%$ & 0.385 \\
\hline & $\begin{array}{c}<10.5 \\
(\mathrm{~g} / \mathrm{dL})\end{array}$ & 84 & $73.4 \%$ & & 69 & $70.4 \%$ & & 15 & $86.7 \%$ & \\
\hline \multirow[t]{2}{*}{ WBC } & $\begin{array}{c}<15 \mathrm{x} \\
10^{9} / \mathrm{L}\end{array}$ & 355 & $78.8 \%$ & 0.836 & 292 & $80.3 \%$ & 0.938 & 63 & $72.0 \%$ & 0.563 \\
\hline & $\begin{array}{l}\geq 15 \mathrm{x} \\
10^{9} / \mathrm{L}\end{array}$ & 155 & $81.4 \%$ & & 117 & $81.4 \%$ & & 38 & $81.3 \%$ & \\
\hline \multirow[t]{2}{*}{ Lymphocytes } & $\begin{array}{c}\geq 0.6 \mathrm{x} \\
10^{9} / \mathrm{L} \\
\text { and } \geq \\
8 \% \\
\quad \mathrm{WBC}\end{array}$ & 443 & $80.4 \%$ & 0.100 & 363 & $80.7 \%$ & 0.743 & 80 & $79.4 \%$ & 0.032 \\
\hline & $\begin{array}{c}<0.6 \mathrm{x} \\
10^{9} / \mathrm{L} \text { or } \\
<8 \% \\
\quad \mathrm{WBC}\end{array}$ & 67 & $74.3 \%$ & & 46 & $80.3 \%$ & & 21 & $60.5 \%$ & \\
\hline All patients & & 510 & $79.6 \%$ & & 409 & $80.6 \%$ & & 101 & $75.6 \%$ & \\
\hline
\end{tabular}

* $P$ values were derived using log-rank test

$H B$, hemoglobin; $W B C$, white blood cells vincristine, procarbazine, and prednisone (escalated-dose BEACOPP,eBEACOPP) for six courses. The PFS at 2 years was $64 \%$ in PET2-positive patients switched to intensified treatment. These results suggest an improvement in PFS for
Table 3 Kaplan-Maier estimates of PFS from PET2 in patients with absolute monocyte counts (AMC) available

\begin{tabular}{lllll}
\hline & & $N$ & $24-$ month PFS & $P$ \\
\hline AMC & $\leq 0.75 \times 10^{9} / \mathrm{L}$ & 152 & $80.0 \%$ & 0.760 \\
& $>0.75 \times 10^{9} / \mathrm{L}$ & 140 & $81.7 \%$ & \\
NLR & $\leq 6$ & 155 & $82.6 \%$ & 0.260 \\
& $>6$ & 134 & $78.2 \%$ & \\
LMR & $>2.1$ & 119 & $78.5 \%$ & 0.664 \\
& $\leq 2.1$ & 169 & $82.5 \%$ & \\
All patients with available monocyte count & & 292 & $80.8 \%$ & \\
\hline
\end{tabular}

* $P$ values were derived using log-rank test

$A M C$, absolute monocyte count; $N L R$, neutrophil lymphocyte ratio; $L M R$, lymphocyte monocyte ratio 
PET2-positive patients compared with the historical experience with continued ABVD. The risk of disease progression for PET2-positive and for IPS high-risk patients were higher in comparison with PET2-negative and IPS low-risk patients, but differences were not statistically significant $(P=0.0442$ and $P=0.2191$, respectively)

As expected, patients treated with eBEACOPP had much more grade 4 and 5 adverse events than those who received ABVD and three treatment-related death were observed, two (4\%) in the eBEACOPP and one $(0.4 \%)$ in the ABVD arm. Further, six patients developed secondary malignancies, including three $(1 \%)$ patients receiving ABVD and three $(6.1 \%)$ in the eBEACOPP arm.

Of note, 58 PET2-negative patients experienced treatment failure, thus demonstrating that PET2 is not a completely reliable test.

In another PET-guided treatment trial, Johnson et al. [9] evaluated 1214 patients with advanced HL. After 2 ABVD courses, PET2-negative patients were randomized to receive 4 cycles of ABVD versus 4 courses of AVD. PET2-positive patients were randomized to receive BEACOPP 14 or eBEACOPP. PFS at 3 years for PET2-positive patients was $67.5 \%$. An analysis of possible predictors of treatment failure was done only in subgroup of patients who had PET2negative scans. Initial Ann Arbor stage was associated with the risk of disease progression, and the differences among stage II versus III versus IV were statistically significant. Although less strong, similar results were observed for IPS.

Any grade 3 or 4 adverse events were reported in $69 \%$ of patients who received $6 \mathrm{ABVD}$, in $65 \%$ of patients switched on AVD, and $80-83 \%$ of patients in BEACOPP arms.

Overall, 19 (4.0\%), 17 (3.6\%), and 22 (12.8\%) patients died in the ABVD, AVD, and BEACOPP arm, respectively. Further, 13 (2.8\%), 11 (2.4\%), and 3 (1.74\%) developed second cancer in the ABVD, AVD, and BEACOPP arm, respectively.

The 3-years PFS in PET2-negative patients was $84.9 \%$ thus confirming that continuing treatment with 4 additional ABVD does not guarantee that patients have been cured.

The results of the GITIL/FIL HD 0607, another PETguided trial with a treatment schema similar to those of the US Intergroup and Johnson trials, have been recently published [10]. The 3-year PFS of PET2-positive patients assigned to BEACOPP, with or without rituximab, was $60 \%$. Evaluating predictive factors of outcome, Gallamini et al. found that by multivariate analysis, IPS was a predictive factor for a positive PET2 scan and for PFS, but not for overall survival.

The most frequent toxicity was hematological, and it was observed in $30 \%$ and $76 \%$ of patients in ABVD and BEACOPP arm, respectively.

Among patients with PET2-positive and PET2-negative results, $16(11 \%)$ and $12(2 \%)$ died respectively.
The 3-year PFS in PET2-negative patients was $82 \%$. In fact, a non-negligible proportion suffered disease recurrence, confirming that PET2 is not a perfect technique in predicting survival outcome.

In a retrospective study performed at a single institution [11], the prognostic meaning of the ratio among lymphocytes, neutrophils, and monocytes was evaluated in newly diagnosed patients with HL treated upfront with a PET2 risk-adapted strategy, switching PET2-positive patients from ABVD to the BEACOPP regimen for 8 cycles. PET2-positive patients who were switched to BEACOPP had a PFS at 5 years of $40.1 \%$. The IPS was unable to provide any clear distinction in the outcomes for PFS, while NLR was prognostic for PFS in univariate and, barely, in multivariate analysis.

Taken together, these results confirm that in the modern era $[2,3]$, and particularly when the treatments were driven by the results of PET2 [8-10], the IPS loses much of its prognostic significance, by reducing the risk of progression in PET2positive patients. In all aforementioned studies [8-10], however, the intensification of therapy in PET2-positive patients was made up with BEACOPP. Here, we evaluate the prognostic significance of IPS in the PET-guided HD0801 trial. By thoroughly analyzing the data, we observed that the IPS completely loses its prognostic value together with all the single parameters that contribute to the IPS. Neutrophils, monocytes, lymphocytes, and the ratio among them also no longer had any predictive value. A possible explanation for the complete loss of meaning of the classic prognostic factors such as IPS, AMC, LMR, and NLR could be the different therapy intensification programs. While in all the aforementioned studies, the intensification was done with BEACOPP, in the HD0801 trial, the intensification was performed by treating PET2-positive patients with 4 cycles of ifosfamide, gemcitabine, and vinorelbine (IGEV) followed by carmustine, cytarabine, etoposide, and melphalan (BEAM)-conditioned autologous bone marrow transplantation. The final results of the phase II part of the HD 0801 study showed that in PET2positive patients, the 2-year PFS increased from $12 \%$ in the historical control to $74 \%$, a slightly higher percentage than those reported in other clinical trials after intensification with BEACOPP, ranging from 60 to $67.5 \%$ [8-10]. While these results were obtained in trials with different enrollment criteria, although all were PET-guided, it could be hypothesized that early transplantation acts more thoroughly than BEACOPP in patients with partial remission or stable disease after 2 cycles of ABVD. The substantial improvement in survival outcomes in PET2-positive patients could therefore explain the complete disappearance of the residual prognostic significance of the IPS at the time of PET-guided therapies.However, despite the improvements in survival outcomes observed in the HD0801 study, PET2-negative and PET2-positive patients still had a PFS of $81 \%$ and $74 \%$, respectively. Therefore, a considerable fraction of patients 
remains at risk of relapse. In brief, the results obtained in the four clinical trials are not substantially different and show PFS between 81 and $85.7 \%$ in PET2-negative patients and between $60 \%$ and $74 \%$ in PET2-positive patients [5, 8-10]. Therefore, while a PET2-adapted strategy can improve survival outcome in PET2-positive patients, a non-negligible proportion of about $20 \%$ of PET2-negative patients remains at risk of relapse. Some patients PET2-negative can progress or relapse as it happens to patients with follicular lymphoma that almost inexorably, after a complete response (CR) will undergo a relapse, showing that the metabolic response does not always correspond to the cure of the disease. Furthermore, it is well established that a proportion of PET2-positive patients can obtain $\mathrm{CR}$ while continuing on ABVD. Inflammation and tumor necrosis can cause false positive interpretation of PET scan. Therefore, if IPS is no longer able to predict outcomes at the time of PET-guided treatment and if PET is a powerful tool, but not a perfect one, it is essential to find new prognostic factors. These parameters could be able to avoid over treatment in false PET2positive patients and to recognize PET2-negative patients still at risk of progression/early relapse. Genetic parameters or new biomarkers or simply a technical/interpretive improvement of PET scan are currently being evaluated. The immune-suppressive component in HL microenvironment evaluated by immunohistochemistry has a prognostic role and seems able to improve the prognostic capabilities of PET2 [12]. The gene expression profile has also been proposed to be predictive of treatment failure [13, 14]. However, both immunohistochemical and genetic studies require prospective evaluation. Recently, some metabolic parameters as the valuation of total lesion glycolysis (TLG) and metabolic tumor volume (MTV) [15] showed prognostic value in patients with advanced stage cHL. Further in advanced disease, baseline TLG and MTV were significantly associated with PET2 results and TLG was a robust predictor of treatment failure and disease relapse [16]. The predictive value of baseline MTV has also been showed in patients with early stage cHL [17]. Recently, a gene expression-based score has been proposed to predict PET2 positivity [18]. All these methods offer strong promise for prognostication but require further study and validation. Refining prognostication is crucial as it represents a further step towards personalized medicine, but so far it remains an issue.

Compliance with ethical standards All local ethic committees at each center approved the study protocol and its amendments in accordance with Italian law and in compliance with the Declaration of Helsinki. Patients provided written informed consent before being included in the study.

Conflict of interest All authors declare that they have no conflict of interest
Open Access This article is licensed under a Creative Commons Attribution 4.0 International License, which permits use, sharing, adaptation, distribution and reproduction in any medium or format, as long as you give appropriate credit to the original author(s) and the source, provide a link to the Creative Commons licence, and indicate if changes were made. The images or other third party material in this article are included in the article's Creative Commons licence, unless indicated otherwise in a credit line to the material. If material is not included in the article's Creative Commons licence and your intended use is not permitted by statutory regulation or exceeds the permitted use, you will need to obtain permission directly from the copyright holder. To view a copy of this licence, visit http://creativecommons.org/licenses/by/4.0/.

\section{References}

1. Hasenclever D, Diehl A (1998) prognostic score for advanced Hodgkin's disease. International Prognostic Factors Project on Advanced Hodgkin's Disease. N Engl J Med 339:1506-1514

2. Moccia AA, Donaldson J, Chhanabhai M, Hoskins PJ, Klasa RJ, Savage KJ, Shenkier TN, Slack GW, Skinnider B, Gascoyne RD, Connors JM, Sehn LH (2012) International Prognostic Score in advanced-stage Hodgkin's lymphoma: altered utility in the modern era. J Clin Oncol 30:3383-3388

3. Diefenbach CS, Li H, Hong F, Gordon LI et al (2015) Evaluation of the International Prognostic Score (IPS-7) and a Simpler Prognostic Score (IPS-3) for advanced Hodgkin lymphoma in the modern era. Br J Haematol 171:530-538

4. Gordon LI, Hong F, Fisher RI, Bartlett NL et al (2013) Randomized phase III trial of ABVD versus Stanford V with or without radiation therapy in locally extensive and advanced-stage Hodgkin lymphoma: an intergroup study coordinated by the Eastern Cooperative Oncology Group (E2496). J Clin Oncol 31:684-691

5. Zinzani PL, Broccoli A, Gioia DM, Castagnoli A, Ciccone G, Evangelista A, Santoro A, Ricardi U, Bonfichi M, Brusamolino E, Rossi G, Anastasia A, Zaja F, Vitolo U, Pavone V, Pulsoni A, Rigacci L, Gaidano G, Stelitano C, Salvi F, Rusconi C, Tani M, Freilone R, Pregno P, Borsatti E, Sacchetti GM, Argnani L, Levis A (2016) Interim positron emission tomography response-adapted therapy in advanced-stage Hodgkin lymphoma: final results of the phase II part of the HD0801 Study. J Clin Oncol 34:1376-1385

6. Tadmor T, Bari A, Marcheselli L et al (2015) Absolute monocyte count and lymphocyte-monocyte ratio predict outcome in nodular sclerosis Hodgkin lymphoma: evaluation based on data from 1450 patients. Mayo Clin Proc 90:756-764

7. Marcheselli R, Bari A, Tadmor T, Marcheselli L, Cox MC, Pozzi S, Ferrari A, Baldini L, Gobbi P, Aviv A, Pugliese G, Federico M, Polliack A, Sacchi S (2017) Neutrophil-lymphocyte ratio at diagnosis is an independent prognostic factor in patients with nodular sclerosis Hodgkin lymphoma: results of a large multicenter study involving 990 patients. Hematol Oncol 35:561-566

8. Press OW, Li H, Schöder H, Straus DJ, Moskowitz CH, LeBlanc M, Rimsza LM, Bartlett NL, Evens AM, Mittra ES, LaCasce A, Sweetenham JW, Barr PM, Fanale MA, Knopp MV, Noy A, Hsi ED, Cook JR, Lechowicz MJ, Gascoyne RD, Leonard JP, Kahl BS, Cheson BD, Fisher RI, Friedberg JW (2016) Using early interim fluorodeoxyglucose-positron emission tomography Imaging: Southwest Oncology Group S0816. J Clin Oncol 34:2020-2027

9. Johnson P, Federico M, Kirkwood A et al (2016) Adapted treatment guided by interim PET-CT scan in advanced Hodgkin's lymphoma. N Engl J Med 23:2419-2429

10. Gallamini A, Tarella C, Viviani S et al (2018) Early chemotherapy intensification with escalated BEACOPP in patients with advanced- 
stage Hodgkin lymphoma with a positive interim positron emission tomography/computed tomography scan after two ABVD cycles: long-term results of the GITIL/FIL HD 0607 Trial. Clin Oncol 36: $454-462$

11. Romano A, Parrinello NL, Vetro C et al (2018) Prognostic meaning of neutrophil to lymphocyte ratio (NLR) and lymphocyte to monocyte ration (LMR) in newly diagnosed Hodgkin lymphoma patients treated upfront with a PET-2 based strategy. Ann Hematol 97: 1009-1018

12. Agostinelli C, Gallamini A, Stracqualursi L et al (2016) The combined role of biomarkers and interim PET scan in prediction of treatment outcome in classical Hodgkin's lymphoma: a retrospective, European, multicentre cohort study. Lancet Haematol 10: e467-e479

13. Steidl C, Lee T, Shah SP et al (2010) Tumor-associated macrophages and survival in classic Hodgkin's lymphoma. N Engl J Med 362:875-885

14. Scott DW, Chan FC, Hong F et al (2013) Gene expression-based model using formalin-fixed paraffin-embedded biopsies predicts overall survival in advanced-stage classical Hodgkin lymphoma. J Clin Oncol 31:692-700

15. Kanoun S, Rossi C, Berriolo-Riedinger A et al (2014) Baseline metabolic tumour volume is an independent prognostic factor in Hodgkin lymphoma. Eur J Nucl Med Mol Imaging 41: 1735-1743

16. Pike LC, Kirkwood AA, Patrick P et al (2017) Can baseline pet-ct features predict outcomes in advanced Hodgkin lymphoma? A prospective evaluation of UK patients in RATHL trial (cruk /07/033). International Conference on Malignant Lymphoma Palazzo dei Congressi, Lugano (Switzerland), 14 - 17 June, 2017. Hematol Oncol 35:37-38

17. Cottereau AS, Versari A, Loft A, Casasnovas O, Bellei M, Ricci R, Bardet S, Castagnoli A, Brice P, Raemaekers J, Deau B, Fortpied C, Raveloarivahy T, Van Zele E, Chartier L, Vander Borght T, Federico M, Hutchings M, Ricardi U, Andre M and Meignan M (2018) Prognostic value of baseline metabolic tumor volume in early-stage hodgkin lymphoma in the standard arm of the H10 trial. Blood 131:14561463

18. Donati B, Casali M, Fama A et Al. A gene expression-based score to predict interim PET positivity in Hodgkin lymphoma patients treated with ABVD. IHCL 2019 Abstract book n. 17: p48

Publisher's note Springer Nature remains neutral with regard to jurisdictional claims in published maps and institutional affiliations. 